\title{
A New Approach to Survivability of Connection Oriented Networks
}

\author{
Krzysztof Walkowiak \\ Chair of Systems and Computer Networks, Faculty of Electronics, Wroclaw University of \\ Technology, Wybrzeze Wyspianskiego 27, 50-370 Wroclaw, Poland \\ walkowiak@zssk.pwr.wroc.pl
}

\begin{abstract}
Issues of network reliability are very important in modern computer networks. Even a short failure of a network link can cause substantial consequences. In this paper we concentrate on survivability of connection oriented networks (ATM, MPLS, Frame Relay). We introduce a new approach to network survivability. We propose a novel function for optimization of network routes. A combinatorial problem is formulated and solved by an heuristic algorithm. Numerical results of simulations and discussion are included.
\end{abstract}

\section{Introduction}

Nowadays many organizations, institutions, companies use computer networks as a primary medium for transmitting various kinds of information. Telecommunication companies and operators focus on new ideas and concepts in order to enable radical transformation of networks and service infrastructures. Therefore a new multiservice ubiquitous architecture with unified control and management called Next Generation Network (NGN) is developed to overcome most of problems now being encountered by major players of the telecommunication world. Loss of services or flow in highspeed fiber systems could cause a lot of damages including economic loses, significant revenue loss, political conflicts, human health. Since NGN networks are expected to offer many various services with QoS guarantees, it is a key issue to introduce some survivability mechanisms enabling the network to work in spite of failures. Therefore engineers and network companies deploy self-healing restoration methods to provide network survivability.

For the context of this paper we concentrate on connection oriented networks. Major examples of connection oriented technologies are ATM (Asynchronous Transfer Mode), MPLS (Multiprotocol Label Switching), Frame Relay.

In this work we introduce a new approach to network survivability. We develop a new function for optimization of network routes. Next we formulate a combinatorial problem and propose an heuristic algorithm solving this problem. Numerical results of a range of simulations are included. 


\section{Survivability of Connection Oriented Networks}

For provision of network survivability two methods can be applied: protection or restoration. The distinction between protection and restoration is connected with the different time scale in which they operate. Protection needs preallocated network resources while restoration applies dynamic resource establishment.

Connection oriented technologies (ATM, MPLS) uses similar approach to enable network survivability. The main idea of this approach is as follows. Each connection, i.e. virtual path in ATM or label switched path in MPLS, has a primary route and a backup route. The primary route is used for transmitting of data in normal, failure-free state of the network. After a failure of the primary route, the failed path is switched to the backup route. The process of switching is easy, i.e. the connection's identifier numbers are changed in network nodes. All backup paths have zero bandwidth, after activation there are assigned with necessary bandwidth. The configuration of backup routes can be found by special algorithms and loaded to network nodes.

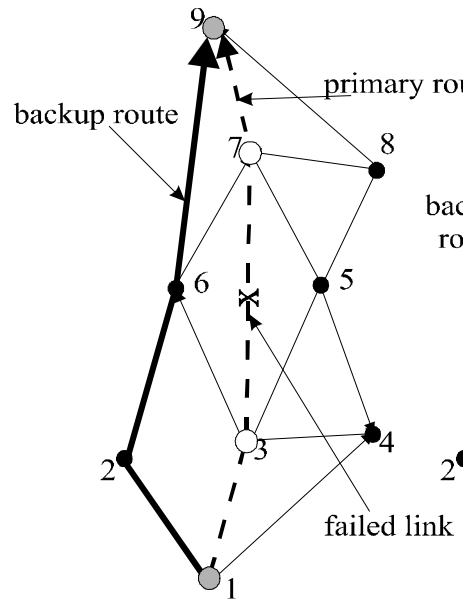

(a)

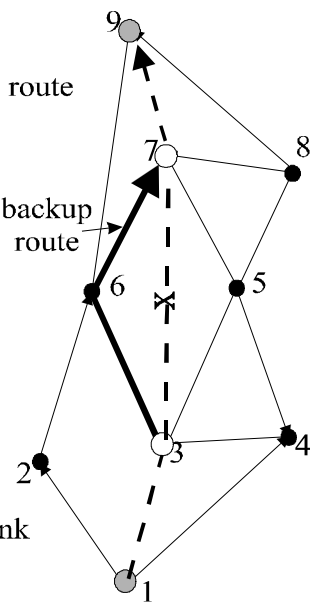

(b)

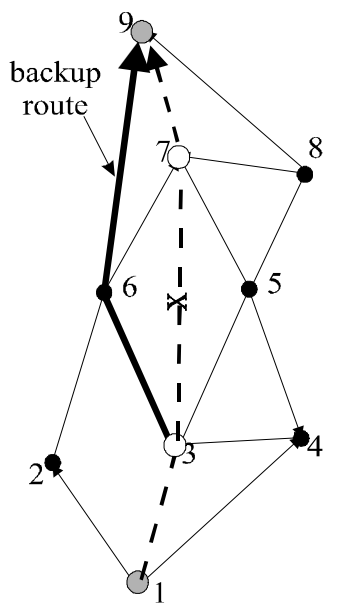

(c)

Fig. 1. Rerouting strategies: (a) Source-based rerouting. (b) Local rerouting. (c) Localdestination rerouting

Three rerouting strategies for ATM networks are proposed in [1], [2]:

- Source-Based Rerouting (SBR). Each connection affected by a network failure is traced back to its source node, which is responsible for rerouting of the connection on a backup route, link-disjoint with the primary route. (Fig. 1a)

- Local Rerouting (LR). The backup route is found only around the failed link. The upstream node of the failed link is responsible for rerouting. (Fig. 1b)

- Local-Destination Rerouting (LDR). This strategy is a compromise of local and source-based rerouting. The backup route is disjoint with the primary route starting 
from the upstream node of the failed link. That node is responsible for rerouting. (Fig. 1c)

Figure 1 illustrates presented rerouting strategies. Path with primary route 1-3-7-9 is broken when the link 3-7 fails. For source-based rerouting the node 1 is informed about the failure and is responsible for switching to a backup path 1-2-6-9 (Fig. 1a). In local rerouting scheme, the node 3 switches to a backup path 3-6-7 to omit the failed link (fig. 1b). It results in a backup route 1-3-6-7-9 for demand pair 1-9. Finally, for local-destination rerouting, node 3 switches the path to a 3-6-9 route, since the path is not changed from source node to the beginning node of failed link, it yields a 1-3-6-9 backup route (Fig. 1c). If we choose for local rerouting strategy a 3-6-9-7 path to omit the 3-7 link, the considered virtual path suffers backhauling, as two extra hopes are traversed (from node 9 to node 7 and back from node 7 to node 9) [1]. Configurations of working and recovery LSPs used for rerouting and protection switching in MPLS networks are similar to ATM rerouting strategies presented above [9].

In fiber networks a single-link failure is the most common and frequently reported failure event [8]. Therefore, in most of optimization models a single-link failure is consider as the basic occurrence. Spare capacity is computed to provide full restoration in case of a failure of any single-link. However, backup routes can be applied in networks with limited resources (capacity of links). In such networks, $100 \%$ restoration is not always possible and routes are designed to minimize effects of the failure. Authors of [8] propose to use two-step restoration, i.e upon a failure the fast restoration is executed in order to accelerate a recovery from the failure. Next the assignment of connections is newly calculated in order to prepare the network for further failures. More details on the topic of ATM and MPLS survivability can be found in [1], [2], [7], [8], [9], [10].

\section{Definition of a New Function for Rerouting}

We consider an existing facility network, i.e. we do not consider facility capacity planning and topological design. In the reminder of this paper we assume that estimated bandwidth requirements of connections and capacity of all links are given. We apply the local-destination rerouting strategy discussed in previous section. The problem of survivable network design consists in assignment of primary and backup routes in order to minimize the lost traffic due to a failure of any single link. Joint optimization of primary and backup routes is a very complex and an NP-complete problem. The central idea of our approach is to partition the problem into two simpler problems and first optimize primary routes and then backup routes for already established primary routes. Unquestionably, independent optimization of primary and backup routes cannot yield an optimal solution, but obtained results proves robustness of this approach [11]. A significant issue is to develop an objective function for primary routes' assignment problem. Such function must indicate preparation of the network to the restoration process. This section is devoted to definition of a new function for localdestination rerouting. When the primary routes are found, for assignment of backup 
routes the function of lost flow due to a failure of a single link can be used. Some previous authors have introduced similar approach for local rerouting strategy in [8] and formulated a problem of primary routes assignment with the objective function using the $k$-shortest path based rerouting.

We model connection oriented network as a directed graph $G=(N, L, C)$ where $N$ is a set of $n$ nodes (vertices) representing network switches, $L$ is a set of $l$ links (directed edges) and $C$ is a vector of link capacity. In our approach we assume that traffic of various connections is deterministically multiplexed. Therefore bandwidth of various connections can be simply summed to check capacity constraints. The notion of equivalent capacity, proposed in paper [5], provides a unified metric representing the load for the connections, and can be applied for determining the estimated bandwidth requirement for connections. This approach simplifies the analysis.

To analyze properties of the local-destination rerouting let's consider a node $w \in N$, that has $n_{w}$ leaving links numbered from 1 to $n_{w}$. To mathematically represent the problem, we introduce the following notations

$f_{1}, f_{2}, \ldots, f_{n_{w}} \quad$ Flow of links leaving the node $w$.

$c_{1}, c_{2}, \ldots, c_{n_{w}} \quad$ Capacity of links leaving the node $w$.

$F^{w}=\sum_{i=1}^{n_{w}} f_{i} \quad$ Flow leaving the node $w$ (flow of all links leaving the node $w$ ).

$C^{w}=\sum_{i=1}^{n_{w}} c_{i} \quad$ Capacity of the node $w$ (capacity of all links leaving the node $w$ ).

We define residual capacity of a link as a difference between capacity and flow of that link.

For the sake of simplicity we introduce the following function

$$
\operatorname{pgt}(x)=\left\{\begin{array}{lll}
0 & \text { for } & x \leq 0 \\
x & \text { for } & x>0
\end{array}\right.
$$

We assume failure of a link $k\left(1 \leq k \leq n_{w}\right)$ leaving the node $w$. In local-destination rerouting flow of the link $k$ must be restored using only other links that leaves the node $w$. Therefore residual capacity of those links is a potential bottleneck of the restoration process. Notice that if

$$
f_{k} \leq \sum_{i=1, i \neq k}^{n_{w}}\left(c_{i}-f_{i}\right)
$$

then flow of the failed link $k$ can be restored using residual capacity of other links leaving the beginning node of the link $k$. Recalling definition of $F^{w}$ and $C^{w}$ we can reformulate (2) in the following way

$$
F^{w} \leq C^{w}-c_{k}
$$

Otherwise if 


$$
f_{k}>\sum_{i=1, i \neq k}^{n_{w}}\left(c_{i}-f_{i}\right)
$$

then some flow of the failed link $k$ can not be restored, because residual capacity of other links leaving the beginning node of the link $k$ is to small. It means that those links block the $100 \%$ restoration and some flow is lost in the node $w$. We define the flow of the link $k$ lost in the node $w$ as flow that cannot be restored using other links leaving the beginning node of the link $k$ due to limited resources of residual capacity in the following way

$$
\operatorname{LFN}_{k}^{w}\left(F^{w}\right)=\operatorname{pgt}\left(F^{w}-\left(C^{w}-c_{k}\right)\right)
$$

Value of the $L F N_{k}^{w}$ function depends on the flow $F^{w}$ leaving the node $w$, it is not dependent directly on the flow $c_{k}$ of the link $k$. Accordingly we formulate the function $L F N^{w}:\left[0 ; C^{w}\right] \rightarrow\left[0 ; C^{w}\right]$ of lost flow in the node $w$ as a sum over all links leaving that node

$$
\operatorname{LFN}^{w}\left(F^{w}\right)=\sum_{i=1}^{n_{w}} \operatorname{pgt}\left(F^{w}-\left(C^{w}-c_{i}\right)\right)
$$

Without loss of generality, we assume that

$$
c_{1} \geq c_{2} \geq \ldots . \geq c_{n_{w}}
$$

The function $\operatorname{LFN}^{w}\left(F^{w}\right)$ is a piece-wise linear function. In case $0 \leq F^{w} \leq C^{w}-c_{1}$ the function has a value of 0 , since for each link leaving the considered node $w$ the flow is not blocked by residual capacity of other links leaving that node. If $C^{w}-c_{1}<F^{w} \leq C^{w}-c_{2}$ only flow of the link number 1 cannot be restored in $100 \%$ and part of this flow is lost in node $w$. If we continue this consideration we may reformulate function (6) as follows

$$
L F N^{w}\left(F^{w}\right)=\left\{\begin{array}{ccc}
0 & \text { for } & 0 \leq F^{w} \leq C^{w}-c_{1} \\
F^{w}+c_{1}-C^{w} & \text { for } & C^{w}-c_{1} \leq F^{w}<C^{w}-c_{2} \\
2 F^{w}+\left(c_{1}+c_{2}\right)-2 C^{w} & \text { for } & C^{w}-c_{2} \leq F^{w}<C^{w}-c_{3} \\
\cdots & & \\
k F^{w}+\sum_{i=1}^{k} c_{i}-k C^{w} & \text { for } & C^{w}-c_{k} \leq F^{w}<C^{w}-c_{k+1} \\
\cdots & & \\
n_{w} F^{w}+\left(1-n_{w}\right) C^{w} & \text { for } & C^{w}-c_{n_{w}} \leq F^{w} \leq C^{w}
\end{array}\right.
$$

The function $\operatorname{LFN}^{w}\left(F^{w}\right)$ is a convex function for $F^{w} \in\left[0 ; C^{w}\right]$. The formal proof of can be found in [11]. 


\section{Optimization Problem of Primary Routes' Assignment}

In this section we will formulate the optimization problem of primary routes' assignment with the objective function $L F N$ (8). Without loss of generality we assume that sets containing proposals of primary routes that conform the LDR strategy are known. The hop-limit approach proposed in [6] can be used in order to calculate such sets. It means that we do not process all possible routes, but only a subset of them. It results in a big reduction of the optimization problem size.

To mathematically represent the problem we introduce the following notations

$P \quad$ Set of $p$ virtual paths in the network.

$Q_{i} \quad$ Estimated bandwidth requirement for connection $i$.

$\Pi_{i} \quad$ Set of working routes for connection $i \Pi_{i}=\left\{\pi_{i}^{k}: k=1, \ldots, l_{i}\right\}$.

$x_{i}^{k}=\left\{\begin{array}{l}1, \text { if } \pi_{i}^{k} \text { is the primary route for connection } i . \\ 0, \text { otherwise. }\end{array}\right.$

$a_{i j}^{k}=\left\{\begin{array}{l}1, \text { if the primary route } \pi_{i}^{k} \text { uses link } j \in L . \\ 0, \text { otherwise. }\end{array}\right.$

$X_{r} \quad$ Set of all variables $x_{i}^{k}$ equal to 1 .

Using the function (6) we define the objective function for the problem of primary routes assignment (PRA) for local-destination rerouting as a sum over all links or all nodes in the network

$$
\operatorname{LFN}\left(X_{r}\right)=\sum_{w=1}^{n} \sum_{i=1}^{l} u_{i}^{w} \operatorname{LFN}{ }_{i}^{w}\left(F^{w r}\right)=\sum_{w=1}^{n} L F N^{w}\left(F^{w r}\right)
$$

where $u_{i}^{w}$ is binary variable that is equal to 1 if node $w$ is the beginning node of link $i$ and is equal to 0 otherwise. We consider a single failure of any link and assume that probability of such a failure is the same for all links, therefore we don not introduce the probability to the function (9). Generally, the function $L F N$ (9) represents the preparation of the network using the LDR strategy to the restoration process after a failure of any single link. As mentioned in previous section, in the LDR strategy the beginning node of the failed link is a bottleneck of the restoration. Therefore it is significant to correctly assign flows of links leaving that node.

The PRA optimization problem can be formulated as follows

$$
\min _{X_{r}} \operatorname{LFN}\left(X_{r}\right)
$$

subject to

$$
\sum_{k=1}^{l_{i}} x_{i}^{k}=1 \quad \text { for } \quad i=1, \ldots, p
$$




$$
\begin{gathered}
x_{i}^{k} \in\{0,1\} \text { for each } i=1, \ldots, p ; k=1, \ldots, l_{i} \\
X_{r}=\left(\underset{i, k: x_{i}^{k}=1}{\bigcup}\left\{x_{i}^{k}\right\}\right) \\
f_{j r}=\sum_{i=1}^{p} \sum_{k=1}^{l_{i}} a_{i j}^{k} x_{i}^{k} Q_{i} \\
F^{w r}=\sum_{m=1}^{l} u_{m}^{w} f_{m r}=\sum_{m=1}^{l} \sum_{i=1}^{p} \sum_{k=1}^{l_{i}} u_{m}^{w} a_{i m}^{k} x_{i}^{k} Q_{i} \\
f_{j r} \leq c_{j} \quad \text { for } \quad j=1, \ldots, l
\end{gathered}
$$

The objective function (10) is a flow lost in any node of the network due to failure of any single link. Condition (11) states that each connection can use only one primary route. Constraint (12) ensures that decision variables are binary ones. Definition (13) says that $X_{r}$ is a set of variables $x_{i}^{k}$, which are equal to one. The set $X_{r}$ is called a selection. Each selection determines the unique set of primary routes. Condition (14) is a definition of a link flow. Correspondingly (15) is a definition of the total flow leaving the node $w$ for the configuration of routes given by $X_{r}$. Finally (16) is a capacity constraint.

The problem (10-16) is NP-complete because this problem is equivalent to the nonbifurcated flow problem which is NP-complete [3]. Summarizing, in the problem PRA (10-16) we must find a feasible selection $X_{r}$ that minimizes the $L F N$ function and satisfies the capacity constraint.

\section{Heuristic Algorithms}

In order to solve the PRA problem (10-16) we have developed an exact algorithm and two heuristic algorithms. For more details on the exact algorithm refer to [11]. In this section we present and discuss a new heuristic algorithm based on the flow deviation algorithm for non-bifurcated flows proposed in [3].

In order to make easier the consideration we introduce the following function

$$
\operatorname{sigma}(x)=\left\{\begin{array}{lll}
0 & \text { for } & x \leq 0 \\
1 & \text { for } & x>0
\end{array}\right.
$$

Without loss of generality we assume that $w$ is the source node of the link $i$. The metric $l_{i r}^{L F N}\left(F^{w r}\right)$ is defined as follows 


$$
l_{i r}^{L F N}\left(F^{w r}\right)=\sum_{j=1}^{n_{w}} \operatorname{sigma}\left(F^{w r}-\left(C^{w}-c_{j}\right)\right)
$$

Note that the $l_{i r}^{L F N}\left(F^{w r}\right)(18)$, is a derivative of the function $L F N^{w}\left(F^{w r}\right)$ (15) for $F^{w r} \in\left[0, C^{w}\right]$ excluding points $F^{w r}=C^{w}-c_{i}$ for $i=1, \ldots, n_{w}$. In these points the function $l_{i r}^{L F N}\left(F^{w r}\right)$ is equal to the left-sided derivative of the function $\operatorname{LFN}^{w}\left(F^{w r}\right)$. Since the metric $l_{i r}^{L F N}\left(F^{w r}\right)$ of link $i$ depends on the flow leaving the node $w$, for all links leaving the node $w$ the metric has the same value.

The problem PRA (10-16) can be solved using the following algorithm.

\section{Algorithm FDP}

Let $X_{1}$ denote a feasible initial solution. In order to find $X_{1}$ we apply an algorithm based on the initial phase of the FD algorithm [3]. Let $\operatorname{LFN}(H)$ denote a value of the $L F N$ function for a selection $H$.

We start with $r:=1$.

Step 1. Find a selection $S R\left(X_{r}\right)$ of variables $x_{i}^{k}$ associated with the shortest routes $\pi_{i}^{k}$ according to the metric $l_{i r}^{L F N}$ calculated for the selection $X_{r}$. Set $i:=1$ and go to step 2 .

Step 2. Let $H:=X_{r}$.

a) Calculate a selection $V$ from the selection $H$ in the following way $V:=\left(H-\left\{x_{i}^{m}\right\}\right) \cup\left\{x_{i}^{k}\right\}$ where $x_{i}^{m} \in H, x_{i}^{k} \in S R\left(X_{r}\right)$. Routes for other connection except connection $i$ remain unchanged.

b) If $V$ is a feasible selection and $\operatorname{LFN}(V)<\operatorname{LFN}(H)$ then set $H:=V$.

c) If $i=p$ the go to step 3. Otherwise set $i:=i+1$ and go to step $2 \mathrm{a}$.

Step 3. If $H=X_{r}$ stop the algorithm, since the solution cannot be improved. Otherwise set $r:=r+1, X_{r}:=H$ and go to step 1 .

The central idea of the FDP algorithm is as follows. We start with a feasible solution $X_{1}$. For each considered selection $X_{r}$ of routes we calculate a selection $S R\left(X_{r}\right)$ containing the shortest routes according to the metric $l_{i r}^{L F N}$ (Step 1). Next we try to improve the solution by deviation of one selected connection to another route (Step 2). The algorithm converges in a finite number of steps, since there are a finite number of non-bifurcated flows. Repetitions of the same flow are impossible due to the stopping condition (Step 3).

The second heuristic algorithm uses the genetic approach. We modified the wellknown genetic algorithm proposed in [4]. More details on this subject are included in [12]. 


\section{Results}

We implemented presented above heuristics algorithms and the exact algorithm in $\mathrm{C}++$ and performed extensive tests over 8 various networks with the number of nodes varying from 10 to 14 . We compared results of heuristic algorithms with optimal results given by the exact algorithm. We made 309 tests. Genetic algorithm gave results only $0.7 \%$ worse than optimal ones, while algorithm based on the FD method gave results $9.8 \%$ worse than optimal ones. For 285 tests genetic algorithm gives the optimal results. Only results of 12 tests are worse $5 \%$ or more than optimal solutions. Flow deviation heuristic produces poorest results.

On the Fig. 2 we can see the performance of algorithms as a function the average link utilization ( $a v l u$ ) of the network. The algorithm based on the FD method gives the best results for networks with small values of the avlu parameter. For more saturated networks performance of this algorithm is much worse. Results of the genetic algorithm generally do not depend on the network saturation.

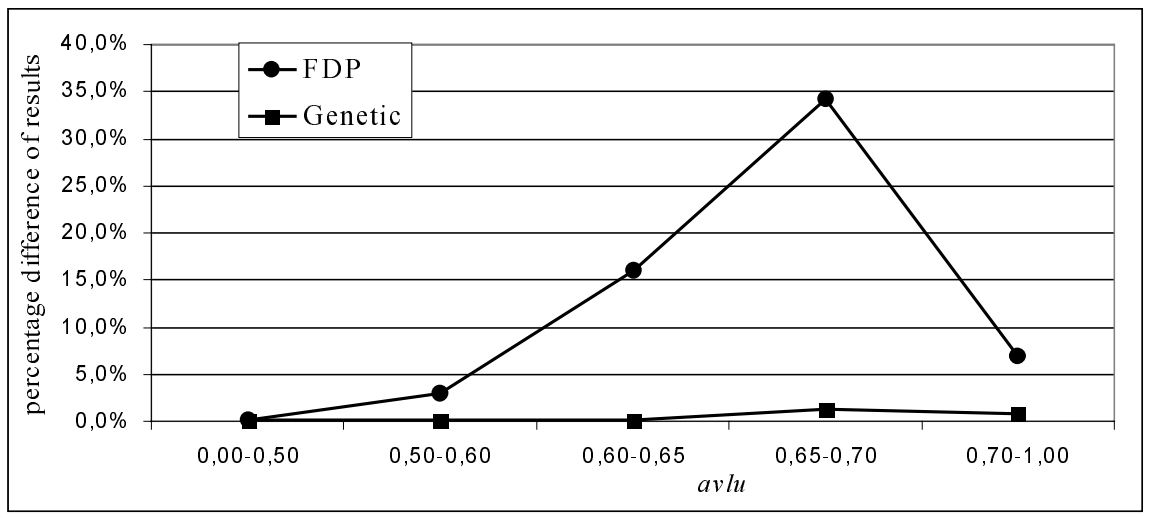

Fig. 2. Graph showing performance of genetic and FDP algorithms comparing to optimal results as a function of the average link utilization

\section{Concluding Remarks}

In this paper we have focused on aspects of survivable network design. We have defined a new objective function $L F N$ for primary routes assignment applying the localdestination rerouting strategy. Next we have formulated an optimization problem PRA of primary routes assignment using the $L F N$ function. To our knowledge this problem has not received much attention in the literature. We have developed new heuristics algorithms for the PRA problem.

We have evaluated the influence of assignment of primary routes using the objective function $L F N$ on the restoration process. As mentioned above, for local rerouting the beginning node of the failed link is responsible for the restoration and is a potential 
bottleneck, in the same way as for local-destination rerouting. For that reason the $L F N$ function can be also applied for assignment of primary routes in local rerouting strategy. We applied as a benchmark the function of lost flow using the k-shortest path (KSP)-based rerouting proposed in [8]. Obtained results presented in [11], [13] confirm the usefulness of the $L F N$ function for effective design of primary routes.

For design of computer networks we can use offline or online algorithms. The function $L F N$ defined above is applicable in both offline and online algorithms for LR and LDR strategies. For offline algorithms the function $L F N$ can be applied as an objective function in the optimization problem as presented in section 5. In online algorithms we can use function $l_{i r}^{L F N}(18)$ as a link metric in order to compute the shortest paths.

The theoretical discussion and experimental results have demonstrated the ability to apply the $L F N$ function for robust design of survivable connection oriented networks.

\section{References}

1. Anderson, J., Doshi, B., Dravida, S., Harshavardhana, P.: Fast Restoration of ATM Networks. IEEE JSAC, 1 (1994) 128-138

2. Ayanoglu, E., Gitlin, R.: Broadband Network Restoration. IEEE Comm. Magazine, 7 (1996) 110-119

3. Fratta, L., Gerla, M., Kleinrock, L.: The Flow Deviation Method: An Approach to Storeand-Forward Communication Network Design. Networks Vol. 3. (1973) 97-133

4. Goldberg, D.: Genetic Algorithms in Search, Optimization, and Machine Learning. Addison-Wesley (1989)

5. Guerin, R., Ahmadi, H., Nagshineh, M.: Equivalent Capacity and Its Application to Bandwidth Allocation in High-Speed Networks, IEEE JSAC, 9 (1991) 968-981

6. Herzberg, M., Bye, S., Utano, A.: The Hop-Limit Approach for Spare-Capacity Assignment in Survivable Networks. IEEE/ACM Transactions on Networking, 6 (1995) 775-784

7. Kawamura, R., Tokizawa, I.: Self-healing Virtual Path Architecture in ATM Networks. IEEE Comm. Magazine, 9 (1995) 72-79

8. Murakami, K., Kim, H.: Virtual Path Routing for Survivable ATM Networks. IEEE/ACM Transactions on Networking, 2 (1996) 22-39

9. Sharma, V., Hellstrand, F. (ed.): Framework for MPLS-based Recovery - Work in Progress, <draft-ietf-mpls-recovery-frmwrk-08.txt> (2002)

10. Veitch, P., Johnson, D.: ATM Network Resilience, IEEE Network, 5 (1997) 26-33

11. Walkowiak, K.: Algorithms for assignment of virtual paths in survivable ATM networks. Ph.D. Thesis, Sci. Papers Division of Systems and Computer Networks of Wroclaw Univ. Technolog., Report Preprinty 2/00 (2000) (in Polish)

12. Walkowiak, K.: Genetic approach to virtual paths assignment in survivable ATM networks, $7^{\text {th }}$ International Conference on Soft Computing MENDEL 2001, Brno (2001) 1318

13. Walkowiak, K.: Assignment of virtual paths in survivable ATM networks for localdestination rerouting. Third International Workshop on Design of Reliable Communication Networks DRCN 2001, Budapest (2001) 273-280 